\title{
Conceptual Overview of Laminar and Turbulent Flows Past Smooth and Rough Circular Cylinders
}

M. M. Zdravkovich, Reader

Department of Aeronautical and Mechanical Engineering, Salford University, U.K.

\section{ABSTRACT}

Flow around a circular cylinder is governed by three transitions: in-the -near-wake, along the free shear layers and along the boundary layers. Each transition is characterized by several flow regimes which are sensitive to disturbances. The variation of mean and fluctuating lift and drag coefficients, friction and pressure coefficients are compiled in the range $10^{\circ}<\operatorname{Re}<10^{8}$ for the disturbance-free flow.

The concept of possible inversion of influencing to governing parameters and vice versa is applied to rough cylinders and turbulent flow. The free stream turbulence and surface roughness affect differently the three transitions and may cause an obliteration of some flow regimes in addition to moving the transition state as a whole to lower Reynolds numbers.

KEYWORDS: Circular cylinder, Flow regimes, Free-stream turbulence, Surface roughness.

\section{CONCEPTUAL INTRODUCTION}

A peculiar feature of flow around all bluff bodies in general and around a circular cylinder in particular is that transition from laminar to turbulent flow does not occur simultaneously in all disturbed regions. Instead, a distinct succession of transition states takes place over an enormous range of Reynolds numbers, see Roshko and Fiszdon(1969).

Each transition state is sensitive to extremely small disturbances, like free stream turbulence, surface roughness and many others. The disturbances can initiate each transition at a lower Reynolds number and can modify or inhibit some flow structures. This means that the Reynolds number is expected to be a single governing parameter only for the disturbance-free flow.

The second peculiar feature of flow around bluff bodies is that the disturbances expressed through the influencing parameters may become the governing parameters in some transition states. When the disturbances exceed a certain value the flow becomes governed by them and not by the Reynolds number. This important feature of the flow around cylinders has not yet been sufficiently appreciated.

Only two kinds of disturbances will be discussed in this review: free stream turbulence and surface roughness. They are the most common disturbances in practical applications.

The free stream turbulence is described by the intensity $T_{i}$, scale $T_{S}$ and frequency spectrum $\mathrm{T}_{f}$ of random three-dimensional velocity fluctuations. It will be shown that the influencing parameters $T_{i}$ and $T_{S}$ affect different transition states in a different way. 
The surface roughness can be characterized by at least two influencing parameters: the relative size of the roughness, $r$, and its texture. Most of the research was carried out by the evenly roughened surface described by the $r / D$ parameter while the uneven surface roughness attracted little attention with the possible exception of tripping wires.

\section{TRANSITION STATES}

The state of flow disturbed by the cylinder may be fully laminar L, a series of transitions $T_{r}$ or fully turbulent $T$. Typical transition states of flow around the circular cylinder are sketched in Fig. 1. The first transition state occurs in the wake, Fig.1a, where laminar vortices become turbulent due to three-dimensional distortions further downstream. The turbulence spreads upstream by the increasing Reynolds number, but the free-shear layers surrounding the nearwake remain laminar.

The second transition state in Fig.1b occurs in the free-shear layers. The transition region gradually moves upstream towards the separation point with increasing Reynolds number.

The third transition around separation, see Fig.1c was historically discovered first on spheres in 1912 and on cylinders by Taylor (1915). This transition produces the largest effect on the drag force. There is an extremely complicated interaction between the separation and transition before the boundary layers become fully turbulent along the separation line.

The fourth and last transition state takes place in the boundary layers away from the separation as depicted in Fig.1d. The upstream movement of the transition region with increasing Reynolds number eventually reaches the stagnation point. Beyond the end of the fourth transition state all regions of the disturbed flow are fully turbulent.

The list of flow states and adopted notation is as follows:

1. L Laminar in all regions of flow

2. TrW Transition in wake, laminar elsewhere

3. TrSL Transition in free shear layers, wake turbulent

4. TrS Transition around separation, boundary layer laminar

5. TrBL Transition in boundary layers

6. $T$ Turbulent in all regions of flow

The states $\operatorname{TrS}$ and $\operatorname{TrBL}$ can be treated as one because separation is the upper limit of the boundary layer.

\section{PROPOSED CLASSIFICATION OF DISTURBANCE-FREE FLOW REGIMES}

The accumulated experimental observations have revealed an enormous variety of regular and irregular flow patterns around circular cylinders. The distinct flow pattern with a limited variation persists only over a range of Reynolds numbers and will be called a flow regime. The flow regimes are expected to be confined within a fixed range of Reynolds numbers only for genuine disturbance - free flows. The uncertainty hidden behind 'genuine' precludes specification of any flow regime by the fixed Reynolds number.

Each state of flow can be subdivided into flow regimes as observed experimentally. The flow regimes are as follows:

L1 - 'creeping' flow (no-separation) $0<\mathrm{Re}<4$ to 5

L2 - steady separated region (closed near-wake) 4 to $5<\mathrm{Re}<30$ to 48

L3 - periodic laminar wake 30 to $40<\mathrm{Re}<150$ to 200

TrW1 - transition of laminar vortices in wake 150 to $200<\operatorname{Re}<200$ to 250 .

TrW2 - transition of vortices during formation 200 to $250<\operatorname{Re}<350$ to 500

TrSL1 - transition waves in free shear layers 350 to $500<\mathrm{Re}<1 \mathrm{k}$ to $2 \mathrm{k}$

TrSL2 - transition vortices in free shear layers $1 \mathrm{k}$ to $2 \mathrm{k}<\mathrm{Re}<20 \mathrm{k}$ to $40 \mathrm{k}$

TrSL3 - fully turbulent shear layers $20 \mathrm{k}$ to $40 \mathrm{k}<\mathrm{Re}<100 \mathrm{k}$ to $200 \mathrm{k}$

TrSO - onset of transition on separation $100 \mathrm{k}$ to $200 \mathrm{k}<\mathrm{Re}<320 \mathrm{k}$ to $340 \mathrm{k}$ 
TrS1 - single separation bubble regime $320 \mathrm{k}$ to $340 \mathrm{k}<\mathrm{Re}<380 \mathrm{k}$ to $400 \mathrm{k}$

TrS2 - two-bubble regime $380 \mathrm{k}$ to $400 \mathrm{k}<\mathrm{Re}<500 \mathrm{k}$ to $1 \mathrm{M}\left(1 \mathrm{M}=10^{6}\right)$

TrS3 - supercritical regime $500 \mathrm{k}$ to $1 \mathrm{M}<\mathrm{Re}<3.5 \mathrm{M}$ to $6 \mathrm{M}$.

TrBL4 - transcritical regime, $3.5 \mathrm{M}$ to $6<\mathrm{Re}<6 \mathrm{M}$ to $8 \mathrm{M}$

$\mathrm{T} 1$ - postcritical regime $\mathrm{Re}>8 \mathrm{M}$

T2 - ultimate regime $\mathrm{Re} \rightarrow \infty$

The variation of the flow pattern in these regimes causes continuous or discontinuous changes of the fluctuating and time-averaged(mean) forces exerted on the cylinder. The mean drag force expressed through the drag coefficient $C_{D}$ consists of the friction drag coefficient $C_{D f}$ and pressure drag coefficient $C_{D P}$. Fig. 2 shows that all three drag coefficients fall steeply with rising Re in the laminar state, (L1 and L2) owing to a more rapid increase of the dynamic pressure $\left(\rho v^{2}\right)$ than viscous resistance $(\alpha \mathrm{V})$. The formation of Karman vortex street in L3 produces a rise in $\mathrm{C}_{\mathrm{DP}}$ and a fluctuating lift coefficient $\mathrm{C}_{\mathrm{L}}{ }^{1}$.

The transition in wake $\operatorname{TrW}$ changes the trend and both $\mathrm{C}_{\mathrm{DP}}$ and $\mathrm{C}_{\mathrm{L}}{ }^{1}$ decrease. The appearance of transition waves in TrSL1 elongates the nearwake until $\mathrm{C}_{\mathrm{Dmin}}$ is reached and $\mathrm{C}_{\mathrm{L}}{ }^{1}$ becomes negligible. The transition vortices in free shear layers shorten the vortex formation region in TrSL2 causing an increase in $C_{D}$ and $\mathrm{C}_{\mathrm{L}}{ }^{1}$ see Gerrard (1966). At the end of TrSL2, $\mathrm{C}_{\mathrm{DF}}$ becomes negligible and $\mathrm{C}_{\mathrm{D}}=\mathrm{C}_{\mathrm{DP}}$. The end of the transition in free shear layers TrSL3 is characterised by the invariance of $C_{D}, C_{L}{ }^{1}$ and $C_{D}{ }^{1}$ due to the short and invariable vortex formation region.

The third transition state affects separation of boundary layers. At first in TrSO the elongation of the vortex formation region causes a decrease in $C_{D}$ and $\mathrm{C}_{\mathrm{L}}{ }^{1}$ with little change in separation point. Then suddenly the separated shear layers are sufficiently turbulent to reattach and form a separation bubble. The bubble forms at first only on one side of the cylinder $\operatorname{TrS1}$, see Bearman (1969). The sudden appearance of the bubble and delayed final turbulent separation up to $140^{\circ}$ causes a discontinuous fall in $C_{D}$ and $C_{L}{ }^{1}$, rise in strouhal number and the appearance of a large mean lift coefficient, $\mathrm{C}_{\mathrm{L}}=1.2$.

At higher Reynolds number in TrS2, the second separation bubble is suddenly formed on the other side of the cylinder. This causes another discontinuous fall in $\mathrm{C}_{\mathrm{D}}, \mathrm{C}_{\mathrm{L}}$ and $\mathrm{C}_{\mathrm{L}}{ }^{1}$ as depicted in $\mathrm{Fig} .2$.

TrS3 is marked by the spanwise disruption and fragmentation of separation bubbles leading to an irregular separation line. The latter seems to be the reason for the cessation of periodic vortex shedding in the supercritical regime. The local drag coefficient varies along the span, as the bubble changes in shape, within a range shown in $\mathrm{Fig}$. 2. The fluctuating lift $\mathrm{C}_{\mathrm{L}}{ }^{1}$ is due to the turbulent fluctuations in the nearwake. At the end of $\operatorname{TrS} 3$, the separation bubbles are fully obliterated along the span but not simultaneously on both sides; hence the reappearance of $\mathrm{C}_{\mathrm{L}}$.

TrBL is characterised by the reappearance of the periodic vortex shedding, as found by Roshko (1961). The transition in boundary layers moves slowly upstream and away from the now straight separation lines. The slow rise in $C_{D}$ and $\mathrm{C}_{\mathrm{L}}{ }^{1}$ is caused by the shortening of the vortex formation region in the transcritical regime.

The final turbulent state of flow is reached when the wake, free shear and boundary layers are fully turbulent. Although no more transitions are expected the postcritical flow regime is not likely to be invariant. The turbulent boundary layers are thinned with a further increase of the Reynolds number. The continuous thinning of the boundary and free shear layers will affect the location of separation and subsequent roll up of free shear layers. This in turn will influence the width of the nearwake and strouhal number, and presumably the length of the vortex formation region and $C_{D}, C_{L}{ }^{1}$ values. This is not known at present for the postcritical regime.

An ultimate flow regime, when Reynolds number tends asymptotically to infinity, has been theoretically postulated by Batchelor (1956). However, real flows at high Reynolds numbers will inevitably bring new influencing parameters before $\operatorname{Re} \rightarrow \infty$. For example compressibility effect for small diameter cylinder. due to high velocity and even a small surface roughness will interfere with extremely thin boundary layers. The cavitation will appear in liquids at high 
(a)

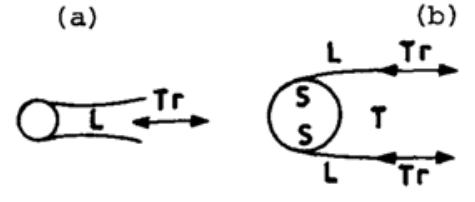

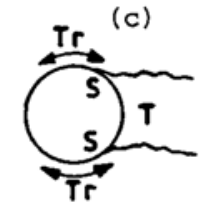

(d)

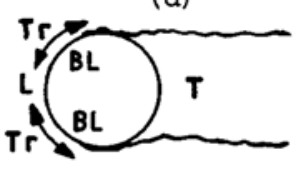

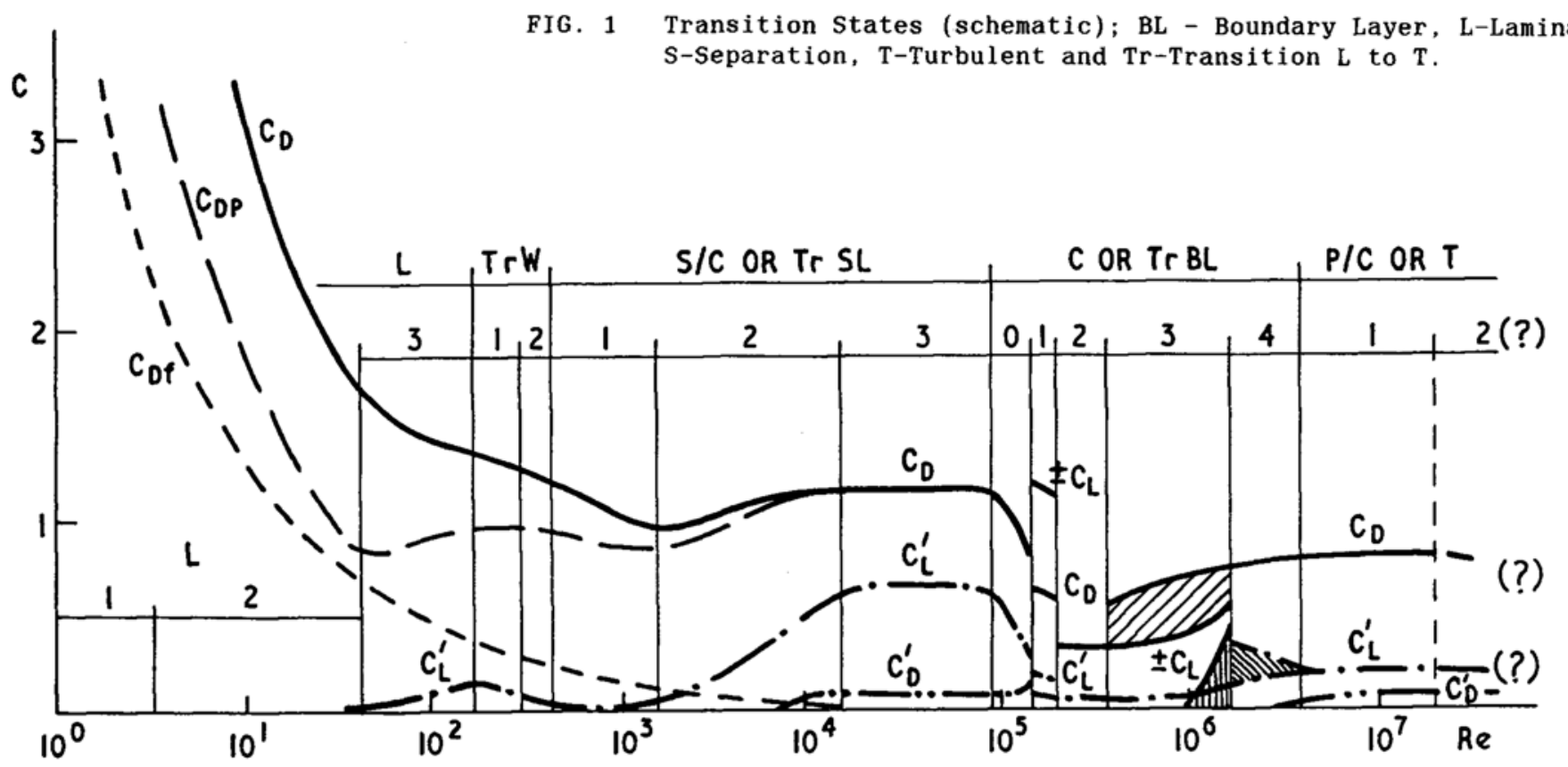

FIG. 2 Force Coefficients versus Reynolds Number; $C_{D}$ - mean drag,

$C_{D f}$ - friction drag, $C_{D P}$ - pressure drag, $C_{D}$ - fluctuating drag, $C_{L}$ - mean lift $C_{L}^{1}$ - fluctuating lift. 
speed. Hence it is doubtful whether the computational $\operatorname{Re} \rightarrow \infty$ flow will be applicable to wind and ocean engineering structures.

\section{EFFECT OF FREE-STREAM TURBULENCE}

The free-stream is usually turbulent in most practical applications but most research has been carried out in almost turbulence-free wind tunnels. However, even small turbulence can affect strongly some transition states. The dominating parameter is the intensity of turbulence $T_{i}$ and only for a few flow regimes the scale $T_{s}$ can become important (see Bearman and Morel, 1983).

The free-stream turbulence has the least effect on the transition in wake. The appearance of irregular spanwise 'fingers' (see Gerrard 1978) initiates the transition by distorting the vortex filaments during their formation. The 'fingers' are located between the laminar free shear layers and can hardly be disturbed by the free stream turbulence; if at all.

Hussain and Ramjee (1976) confirmed that the transition in wake is insensitive to turbulence. Further downstream, however, transition is triggered when velocity fluctuations induced by the decaying laminar vortex filaments become of the same order of magnitude as turbulent fluctuations in the free stream. The higher $T_{i}$ the nearer to the cylinder the transition takes place along the wake.

The free-stream turbulence has the strongest effect on transition in the free shear layers. The three flow regimes in TrSL are affected in a different extent. Least affected is TrSL1 because the laminar transition waves are stable. However, the development of a row of transition vortices in TrSL2 is significantly affected by the turbulence and this in turn shortens the vortex formation region (see Gerrard 1966). Thus TrSL2 can be obliterated by a high $T_{1}$. For example, if $\mathrm{T}_{i}>10 \%$ the drag coefficient reaches 1.2 at $\operatorname{Re}=2 \mathrm{k}$ and $\mathrm{TrSL} 3$ moves in the $10^{3}<\mathrm{Re}<10^{4}$ range.

The upper subcritical regime TrSL3 is characterised with a rapid transition to turbulence in free shear layers. The free stream turbulence has little effect on this type of transition until the transition reaches the separation. This heralds a new transition state TrS.

The transition at separation $\operatorname{TrS}$ is the most strongly affected by the turbulence. Up to three flow regimes in this state can be obliterated by turbulence. The precritical regime TrSO is always triggered earlier by the free stream turbulence. Fig. 3 shows the resemblance in shape of the drag coefficient curves for all turbulent streams and the progressive displacements to lower Reynolds numbers as $T_{i}$ increases. The Strouhal number remains almost constant because the nearwake width is not affected. The turbulence, being a three dimensional phenomenon, reduces the spanwise correlation and extends the length of the vortex formation region. This, in turn, gradually decreases the drag coefficient.

The free stream turbulence has a strong effect on the formation and obliteration of the separation bubbles. The combined effect of three dimensional and randomly modulated fluctuations inhibits the laminar separation and single and two bubble regimes are suppressed. The partial and fragmented formation of separation bubbles on both sides presumably resembles the supercritical regime of the disturbance-free flow around the cylinder. This means that the precritical regimes TrSO is directly followed by the supercritical regime TrS3.

At still higher $T_{i}$, the fragmented separation bubbles are also destroyed. It is expected that the extent of the supercritical regime will gradually shrink until it eventually disappears. The latter has not yet been corroborated by experiments. If this happens then vortex shedding does not cease at all and the precritical regime is succeeded by the transcritical regime TrBL4. The drag coefficient undergoes less and less variation with increasing $T_{i}$, see curves for 4.4\% and 9.1\% in Fig. 3. The Strouhal number reflects these changes in $C_{D}$ and reaches $0.38,0.30$ and 0.24 respectively. Fluctuating lift coefficient $C_{L}{ }^{1}$ rises inversely at $\mathrm{Re}=0.4 \mathrm{M}, \mathrm{C}_{\mathrm{L}}{ }^{1}=0.05,0.08,0.12$ respectively.

It should be pointed out that the effect of the free stream turbulence cannot be thought of as a simple displacement of the transcritical regime to lower Reynolds numbers. This has been noted by Fage and Falkner (1931) by 


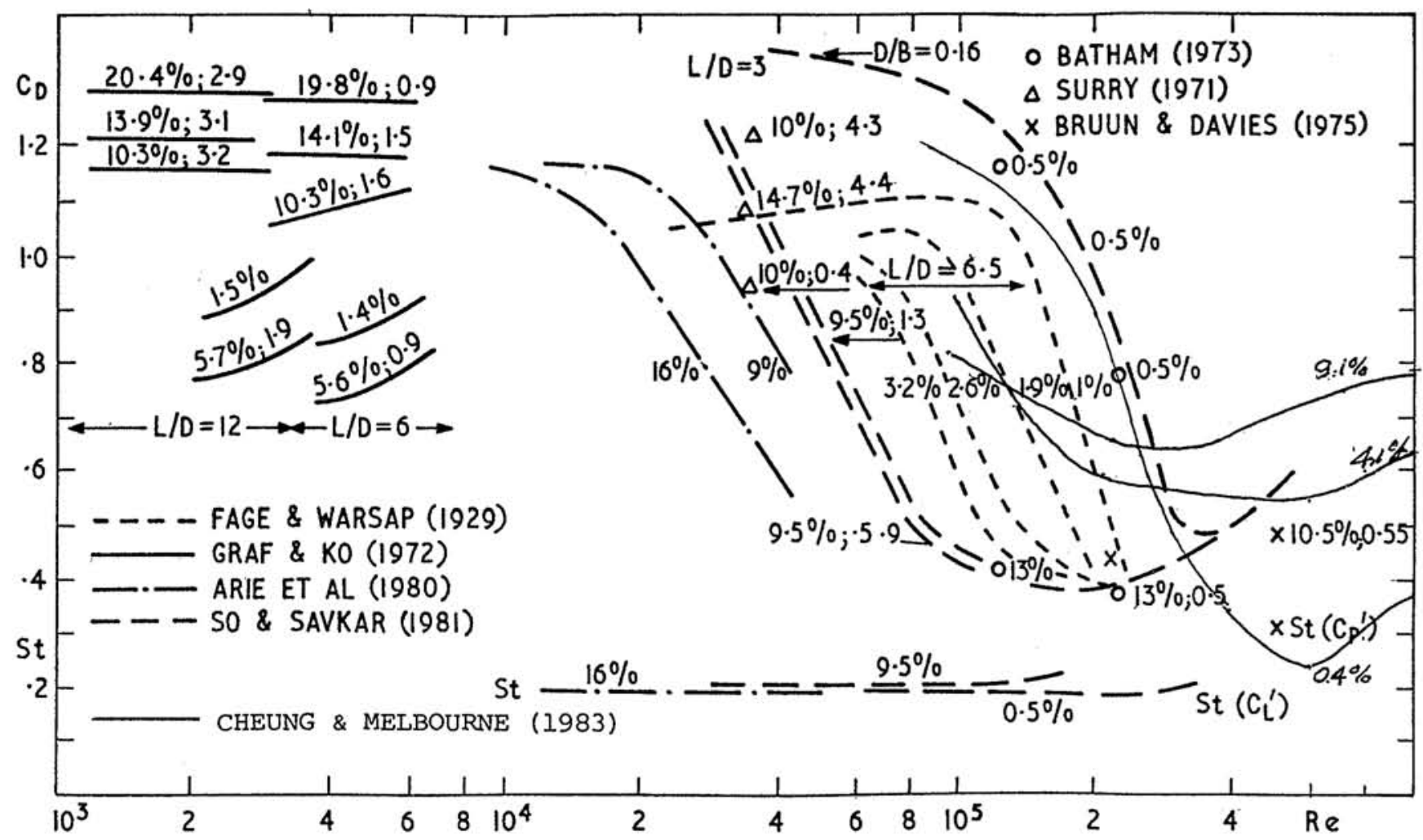

FIG. 3 Drag Coefficient and Strouhal number for Various $T_{i}(\%), L / D-$ aspect ratio and $D / B$ blockage ratio. 
comparing the pressure distribution around the cylinder for the same value of drag coefficient in supercritical and transcritical flow regime. Hence the empirical relationship Re1.94 $\times \mathrm{T}_{i}=0.2 \mathrm{M}$ proposed by Kiya et al (1982) cannot be extended to TrBL4.

\section{EFFECT OF SURFACE ROUGHNESS}

It has been known that thick shear layers in laminar pipe flows are practically insensitive to surface roughness. Similar thick shear layers are formed around a circular cylinder in the laminar state of flow. For example Wieselsberger (1922) measured at $\mathrm{Re}=3$ the thickness of 9D (cylinder diameters). When transition in wake takes places say around $R e=200$, the thickness of the boundary layer is reduced to $0.5 \mathrm{D}$ and the surface roughness still has negligible effect.

The first noticeable effect of the surface roughness on flow takes place in the transition in shear layers or subcritical state. Unlike free stream turbulence that affects directly both boundary and free-shear layers, the surface roughness affects directly only the boundary layers and indirectly through the latter the free shear layers. This important difference means that the surface roughness is less effective in disturbing the free shear layers in TrSL1 and TrSL2. Note that the boundary layer is still $0.12 \mathrm{D}$ at $\mathrm{Re}=1 \mathrm{k}$.

The most powerful influence of the surface roughness occurs in the transition in the boundary layer state since the surface roughness can be regarded as a mechanism which produces local turbulence. Fage and Warsap (1929) documented that even a small surface roughness can trigger the precritical regime prematurely. The stepwise increase in surface roughness caused a systematic change in the shapes and progressive displacement of the drag coefficient curves towards the lower Reynolds numbers. Regretably, Fage and Warsap (1929) did not measure the Strouhal number.

Subsequent measurements of both by Okajima and Nakamura (1973) and Buresti (1980) using sand roughness $S$ and by Achenbach and Heinecke (1981) using pyramidal roughness $\mathrm{P}$, are compiled in Fig. 4 covering the range of relative roughness from 0.0025 to 0.0303 . All minimum drag coefficients and Strouhal numbers curves are connected with vertical lines in order to distinguish and correlate the corresponding curves.

The minimum drag coefficient designates the two bubble regime only for flow past a smooth surface cylinder. This regime is followed by the spanwise fragmentation of bubbles that causes inhibition of vortex shedding in the supercritical regime. The effect of surface roughness is to inhibit the formation of the separation bubble and hence to obliterate one-and two bubble regimes. Achenbach and Heinecke (1981) did not find separation bubbles for the relative roughness greater than 0.003 . The supercritical regimes, denoted by 'No $S t$ ' in Fig. 4, show progressive reduction in extent until at $S .1 / 81$ the supercritical regime disappears. The vortex shedding persists unabated when the relative roughness is greater than 0.01 . This means the precritical regime is followed by the transcritical regime. The latter is also progressively shortened due to the direct effect of surface roughness on the boundary layers from the stagnation point onwards.

The postcritical state of flow is strongly affected not only by the relative magnitude but also by the texture of surface roughness. Fig. 4 shows that the pyramidal roughness obtained by course machining of the surface, $P$ 1/330, produces considerably higher drag coefficient (1.2) than the sand roughness $S$ with spherical elements $(0.75)$. Note that, the other two influencing parameters length to diameter ratio and blockage ratio are almost identical in both experiments.

Karman wrote for pipes "All surfaces may be considered rough when the Reynolds number is sufficiently high; for the frictional resistance of rough walls is made up essentially of the resistances of all excrescences and these become independent of friction at high values of Reynolds number".Typical value of the boundary layer momentum thickness at $90^{\circ}$ in the postcritical state say at $\mathrm{Re}=1 \mathrm{M}$ is $0.002 \mathrm{D}$. Hence all excrescences will protrude when $\mathrm{r} / \mathrm{D}>0.002$. 


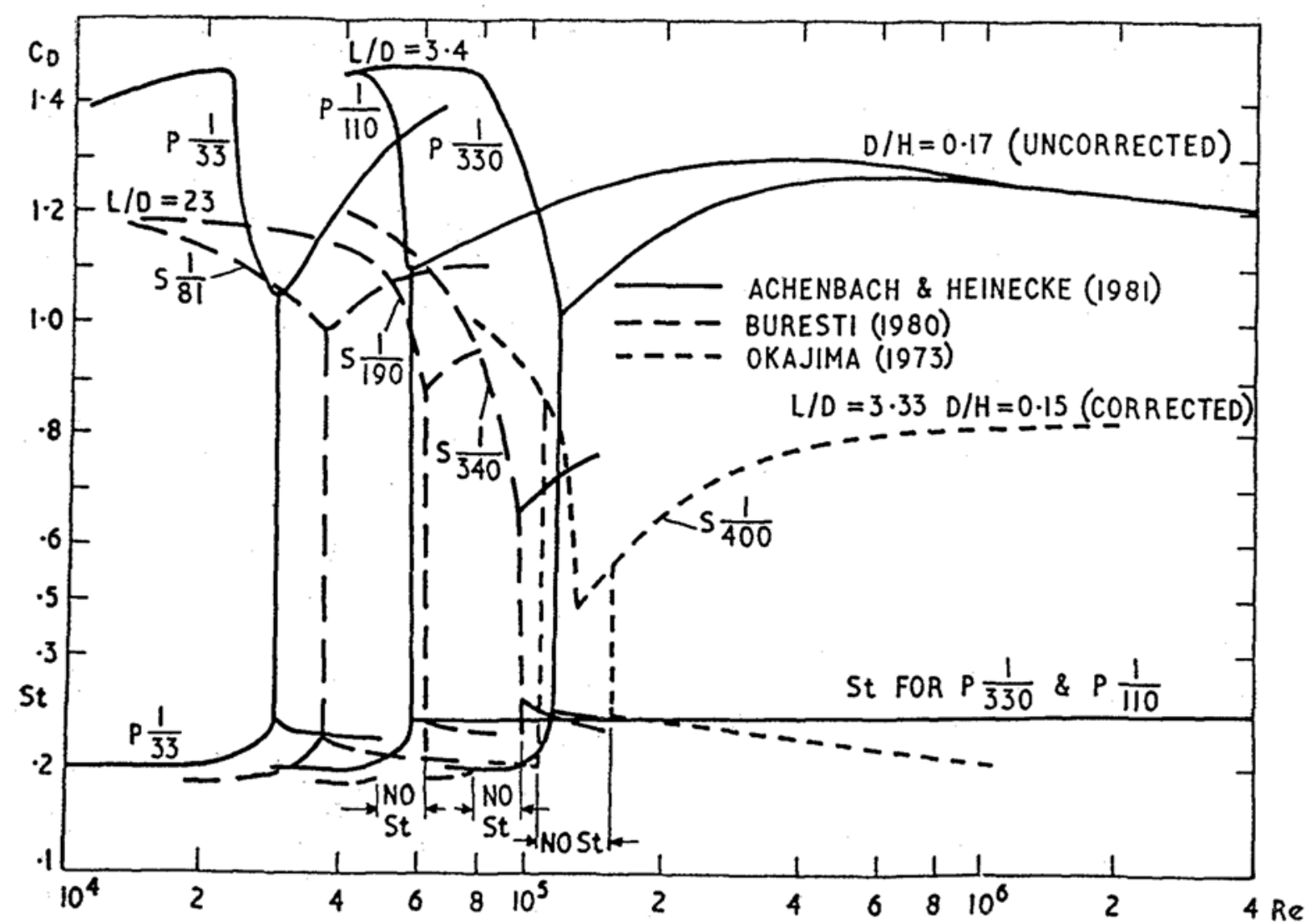

FIG. 4 Drag Coefficient and Strouhal Number for various Relative Roughness Ratio, r/D; L/D - Aspect Ratio, D/H Blockage Ratio, P - Pyramidal Roughness, S - Sand Roughness. 
Nakamura and Tomonari (1982) argue that the roughness Reynolds number based on the size of roughness should be replaced by the empirical correlation $(\mathrm{r} / \mathrm{D})^{0.6}$ Re.

\section{CLOSING REMARKS}

The contribution advanced in this overview is rather conceptual than factual. The well established terms like subcritical, critical and supercritical regimes, originated at times when the knowledge and understanding of flow past cylinder was limited, ought to be reconsidered. The subsequent research shows that the original terminology is inadequate and confusing. The old three regimes are treated now phenomenologically as three transitions: in wake, shear and boundary layers with as many flow regimes in each as required by the observation. The main advantage of the new terminology and classification shows when extended to the disturbed flows. Only two such disturbances are discussed: free stream turbulence and surface roughness. The most important effects of the disturbance is the early initiation of transition and reduction in extent and/or total obliteration of certain flow regimes. The last aspect of the turbulent free-stream and rough cylinder has been ignored in the past and requires further research for a proper quantification of parameters.

It appeared that the most complex flow past circular cylinder is the disturbance-free flow having 15 flow regimes. The disturbances always cause a loss of few flow regimes in the transition states and large disturbances can obliterate a whole transition state. However the postcritical state, when all regions are fully turbulent, shows increasing dependence on the disturbances, which become the governing parameters. The postcritical state is still the least known of all and requires a lot of research.

\section{ACKNOWLEDGEMENT}

This review forms part of the book "Flow around Circular Cylinders" by the author which will be published by Springer Verlag in English (sections 1.1, 3.1, and 6.1). Hence the author reserves the copyright of Figs 1 to 4 .

\section{REFERENCES}

Achenbach, E, 1971, Influence of Surface Roughness on the Crossflow around a Circular Cylinder, Journal of Fluid Mechanics, vol 46, pp 321-335.

Achenbach, E. and Heinecke, E. 1981, On Vortex Shedding from Smooth and Rough Cylinders in the range of Reynolds numbers $6 \times 10^{5}$ to $5 \times 10^{6}$, Journal of Fluid Mechanics, vol. 109, pp 239-251.

Arie, M., Kiya, M., Suzuki, Y., Hagino M., and Takahashi, K., 1981, Characteristics of Circular Cylinders in Turbulent Flows, Bulletin Japan. Soc. Mech Eng., vol. 24, pp 640-647.

Batchelor, G.K. 1956, A Proposal Concerning Laminar Wakes behind Bluff Bodies at Large Reynolds Numbers, Journal Fluid Mechanics, vol 1 , pp. 388 - 398 .

Batham, J.P. 1973, Pressure Distribution on Circular Cylinder at Critical Reynolds Numbers, Journal of Fluid Mechanics, vol. 57 . pp. 209-228.

Bearman, P.W., 1969, On Vortex shedding from a Circular Cylinder in the Critical Regime, Journal of Fluid Mechanics, vol. 37, pp 577-585.

Bearman, P.W. and More1, T. 1983, Effect of Free Stream Turbulence on the Flow around Bluff Bodies, Progress Aerospace Sciences, vol. 20, pp. 97-123.

Bruun, H.H. and Davies, P.O.A.L. 1975. An Experimental Investigation of the Unsteady Pressure Forces on a Circular Cylinder in a Turbulent Cross Flow, Journal Sound and Vibration, vol. 40(4), pp 535-555.

Buresti, G. 1981. The Effect of Surface Roughness on the Flow Regime around Circular Cylinders, Journal of Wind Engineering and Industrial Aerodynamics, vol. 8, pp. 105-114.

Cheung, J.C.K. and Melbourne, W.H. 1983, Turbulence Effects on Some Aerodynamic Parameters of a Circular Cylinder at Supercritical 
Reynolds Numbers, Journal Wind Engineering and Industrial Aerodynamics vol 14, pp. 399-410.

Fage, A. and Warsap, J.H. 1929, The Effects of Turbulence and Surface Roughness on Drag of Circular Cylinders, British Aeronautical Research Council, Rep. \& Memo 1283.

Fage, A. and Falkner, V.M., 1931, Further Experiments on Flow around a Circular Cylinder, British Aeronautical Research Council, Rep. and Memo 1369 .

Farell, C., 1981, Flow around Fixed Circular Cylinders, Fluctuating Loads, Journal Eng. Mech. Div. Amer. Soc. Civ. Eng. vol. 107, No. EM3. pp. 565-598.

Farell, C and Blessman, J. 1982, On Critical Flow around Smooth Circular Cylinders, Journal of Fluid Mechanics, vol. 136, pp $375-391$.

Gerrard, G.H. 1966, The Mechanics of the Formation Region of Vortices behind Bluff Bodies, Journal Fluid Mechanics, vol. 25, pp. 401-413.

Gerrard, J.H. 1978, The Wakes of Cylindrical Bluff Bodies at Low Reynolds Number, Philosophical Transactions Royal Society A, vol. 288 , pp 351-382.

Hussain, A.K.M.F. and Ramjee, V. 1976, Periodic Wake behind a Circular Cylinder at Low Reynolds Numbers, Aeronautical Quaterly vol. 27, pp 123-141.

Kiya, M., Suzuki, Y., Arie, M. and Hagino, M. 1982, A Contribution in the Free Stream Turbulence effect on the Flow past a Circular Cylinder, Journal Fluid Mechanics, vol. 115, pp. $151-164$.

Ko, S.C. and Graf, W.H. 1972, Drag Coefficient of Cylinders in Turbulent Flow, Proc. Am. Soc. Civ. Eng. Journal of Hydraulics Division vol. 98 , No. 485 , pp $897-918$.

Morkovin, M.V. 1964, Flow around Circular Cylinders; a Kaleidoscope of Challenging Fluid Phenomena, Proc. Amer. Soc. Mech. Eng. Symposium on Fully Separated Flows, Philadelphia, pp. 102-112.

Nakamura, Y. and Tomonari, Y. 1982, The Effects of Surface Roughness on the Flow Past Circular Cylinders at High Reynolds Numbers, Journal of Fluid Mechanics, vol. 123, pp 363-378.

Norberg, C. and Sunden, B. 1987, Turbulence and Reynolds Number Effects on the Flow and Fluid Forces on a Single Tube in Cross Flow, Journal of Fluids and Structures, vol. 1, pp. 339-359.

Okajima, A and Nakamura, Y. 1973, Bulletin Research Institute of Applied Mechanics, Kyu Shu University, pp. 387-400 (in Japanese).

Roshko, A. and Fiszdon, W. 1969, On the Persistence of Transition in the Nearwake, Problems of Hydrodynamcs and Continuum Mechanics, Soc. Ind. Appl. Maths, Philadelphia, pp. 606-616.

Roshko, A. 1971, Experiments on Flow Past a Circular Cylinder at very High Reynolds Number, Journal of Fluid Mechanics, vol. 10, pp. $345-356$.

Schewe, G. 1983 , On the Force Fluctuations acting on a Circular Cylinder in Crossflow from Subcritical up to Transcritical Reynolds Numbers. Journal of Fluid Mechanics, vol. 133, pp. 265 - 283.

Schewe, G. 1986, Sensitivity of Transition Phenomena to Small Perturbations in Flow around a Circular Cylinder, Journal of Fluid Mechanics, vol. 172, pp. $33-46$

So, R.M.C. and Savkar, S.D. 1981, Buffeting Forces on Rigid Circular in Cross Flows, Journal Fluid Mechanics, Vol. 105, pp. 397-425.

Surry, D. 1971, Some Effects of Intense Turbulence on the Aerodynamics of a Circular Cylinder at Subcritical Reynolds Number, Journal Fluid Mechanics, vol. 52, pp 543-563.

Taylor, G.I. 1916, Pressure Distribution around the Cylinder, British Advisory Committee Aerodynamics, Rep. \& Memo 191, Also Tech. Rep. vol. Vii, pp 30-31.

Wieselsberger, C. 1922, On the Drag of Circular Cylinders, (in German), Physikalische Zeitschrift, vol. 22, pp. 219-224. 